\title{
Aspirin plus esomeprazole reduced recurrent ulcer bleeding more than clopidogrel in high risk patients
}

Chan FK, Ching JY, Hung LC, et al. Clopidogrel versus aspirin and esomeprazole to prevent recurrent ulcer bleeding. N Engl J Med 2005;352:238-44.

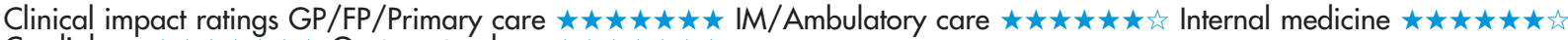

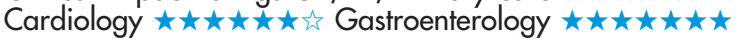

In patients with previous aspirin-induced ulcer bleeding, is clopidogrel non-inferior to low dose aspirin plus esomeprazole for preventing recurrent ulcer bleeding?

\section{METHODS}

$\square$

Design: randomised placebo controlled trial.

$\triangle$

Allocation: concealed.*

Blinding: blinded (clinicians, patients, outcome assessors, \{dato collectors, data analysis, and data safety and monitoring committee $\}+)^{*}$

$\sum$

Follow up period: 12 months.

Setting: Prince of Wales Hospital in Hong Kong

Patients: 320 patients (mean age $72 y, 66 \%$ men) who had a history of ulcer bleeding and endoscopically confirmed ulcer healing and either tested negative for Helicobacter pylori or had successful eradication of $H$ pylori and required antiplatelet therapy during the trial. Exclusion criteria were concomitant use of non-steroidal anti-inflammatory drugs (NSAIDs), cyclooxygenase-2 inhibitors, anticoagulant agents, or corticosteroids; history of gastric surgery (except patch repair); allergy to aspirin or clopidogrel; presence of erosive oesophagitis, gastric-outlet obstruction, terminal illness, or cancer; or requirement for dialysis.

D Intervention: clopidogrel, $75 \mathrm{mg}$, plus placebo $(\mathrm{n}=161)$ or aspirin, $80 \mathrm{mg}$, plus esomeprazole, $20 \mathrm{mg}(\mathrm{n}=159)$ twice daily for 12 months.

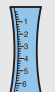

Outcomes: recurrent ulcer bleeding. Secondary outcomes were lower gastrointestinal bleeding and adverse effects.

Patient follow-up: $99 \%$ (intention to treat analysis).

${ }^{*}$ See glossary.

tInformation provided by author.

For correspondence: DrFK $\mathrm{K}$ Chan, Chinese University of Hong Kong, Shati Hong Kong, China. fklchan@cuhk.edu.hk

Source of funding: no external funding.

\section{MAIN RESULTS}

More patients in the clopidogrel group than in the aspirin-plusesomeprazole group had recurrent ulcer bleeding (table). The groups did not differ for lower gastrointestinal bleeding (table). Adverse event rates for clopidogrel and aspirin plus esomeprazole were 1.9\% and $0 \%$, respectively, for extragastrointestinal bleeding; $7.5 \%$ and $2.5 \%$, respectively, for dyspepsia; $5.6 \%$ and $6.9 \%$, respectively, for recurrent ischaemic events; and $1.9 \%$ in each group for allergy.

\section{CONCLUSION}

In patients with previous aspirin induced ulcer bleeding, the addition of esomeprazole to aspirin was better than changing to clopidogrel for reducing recurrent ulcer bleeding.

Abstract and commentary also appear in ACP Journal Club.

\section{Commentary}

Trenter he use of antiplatelet agents (eg, low dose aspirin or clopidogrel) for the prevention of cardiovascular events is widespread. Low dose aspirin is ulcerogenic, so its use alone is contraindicated in patients at high risk of ulcer bleeding. Alternative approaches in such patients include adding a proton-pump inhibitor (PPI) to aspirin ${ }^{1-2}$ or using a nonaspirin agent (clopidogrel) instead. The study by Chan et al is the first to directly compare these 2 approaches in patients who have bled once from an aspirin associated ulcer. Somewhat surprisingly, the results of this study clearly show that the second option is not acceptable. Although clopidogrel had been believed to be non-ulcerogenic, it was associated with a substantially higher incidence of recurrent ulcer bleeding than was low dose aspirin plus a PPI (esomeprazole). Because the incidence of adverse events prevented by antiplatelet therapy was similar in the 2 groups, the recommendation to switch patients with a previous aspirin associated bleeding ulcer to clopidogrel alone is not justified. In patients who truly cannot take aspirin, clopidogrel could be used with a concomitant PPI, although this has not yet been proven to be safe. Walter L Peterson, MD University of Texas Southwestern Medical Center at Dallas Dallas, Texas, USA

1 Lai KC, Lam SK, Chu KM, et al. Lansoprazole for the prevention of recurrences of ulcer complications from long-term low-dose aspirin use. N Engl J Med 2002;346:2033-8.

2 García Rodríguez LA, Ruigómez A. Secondary prevention of upper gastrointestinal bleeding associated with maintenance acid-suppressing treatment in patients with peptic ulcer bleed. Epidemiology 1999:10:228-32.

Clopidogrel $v$ aspirin plus esomeprazole for patients with previous aspirin induced bleeding*

\begin{tabular}{llll}
\hline Outcomes at $\mathbf{1 2}$ months & Clopidogrel & Aspirin + esomeprazole & Difference (95\% CI) \\
\hline Cumulative incidence of recurrent ulcer bleeding & $8.6 \%$ & $0.7 \%$ & $7.9 \%(3.4$ to 12.4$)$ \\
Cumulative incidence of lower gastrointestinal bleeding & $4.6 \%$ & $4.6 \%$ & No difference \\
\hline
\end{tabular}

${ }^{*} \mathrm{Cl}$ defined in glossary 